\title{
Comparison of clinicopathological features and prognosis between IgA nephropathy and purpura nephritis in adults with diffuse endocapillary proliferation: a single-center cohort study
}

\author{
Jin Yu, Xiaoqiu Ni, Jun Ni, Fei Jiang, Dongrong Yu, Liusha Zhou \\ Department of Nephrology (Key Laboratory of Management of Kidney Disease in Zhejiang Province), Guangxing Hospital Affiliated to Zhejiang \\ Chinese Medical University (Hangzhou Hospital of Traditional Chinese Medicine), Hangzhou 310007, China \\ Contributions: (I) Conception and design: J Yu, L Zhou; (II) Administrative support: L Zhou; (III) Provision of study materials or patients: J Yu, X Ni, \\ F Jiang, D Yu, L Zhou; (IV) Collection and assembly of data: J Yu, X Ni, F Jiang, D Yu; (V) Data analysis and interpretation: J Yu; (VI) Manuscript \\ writing: All authors; (VII) Final approval of manuscript: All authors. \\ Correspondence to: Liusha Zhou, MD. Department of Nephrology, Guangxing Hospital Affiliated to Zhejiang Chinese Medical University (Hangzhou \\ Hospital of Traditional Chinese Medicine), No. 453 Tiyuchang Road, Hangzhou 310007, China. Email: liushazhou@sohu.com.
}

Background: Clinical manifestations and histological lesions of IgA nephropathy and Henoch-Schönlein purpura nephritis (HSPN) are different, but related, and are also correlated with the renal outcomes. This study aimed to compare the features of immunoglobulin A nephropathy (IgAN) and HSPN in adult patients with diffuse endocapillary proliferation (DEP) lesions aiming to clarify the differences and relationships in the clinicopathological findings and outcome.

Methods: Twelve patients with DEP-IgAN and 10 patients with DEP-HSPN were enrolled. Twenty four patients with IgAN (NDEP-IgAN) and matched 20 patients with HSPN (NDEP-HSPN) were enrolled at the same ratio (1:2). The clinicopathological features, clinical efficacy, and renal outcomes were analyzed in the four groups.

Results: DEP patients with IgAN or HSPN had worse clinical manifestations (more severe proteinuria, lower serum ALB, higher incidence of gross hematuria). The proteinuria in the DEP-HSPN group was more severe than in the DEP-IgAN group. There was no significant difference in the serum creatinine among four groups. The incidence of endothelial swelling was significantly higher in the DEP-HSPN group than in the NDEP-HSPN group and DEP-IgAN group. The S1 score of Oxford classification was more common in the DEP-IgAN group than in the DEP-HSPN. None in the DEP-IgAN group reached endpoint events during the follow-up period, while the renal outcomes were significantly poorer in the DEP-HSPN group than in the DEP-IgAN and NDEP-HSPN groups. No significant difference was observed in the cumulative renal survival among four groups $\left(\chi^{2}=7.264, \mathrm{P}=0.064\right)$, but patients in the DEP-HSPN group had markedly lower renal cumulative survival rate as compared to the NDEP-HSPN group $\left(\chi^{2}=4.875, \mathrm{P}=0.027\right)$.

Conclusions: The DEP is significantly associated with more severe proteinuria and hematuria regardless the IgAN and HSPN. Among DEP patients, patients with HSPN have poor therapeutic efficacy and renal outcomes, even under active immunosuppressive therapy, as compared to those with IgAN.

Keywords: Diffuse endocapillary proliferation (DEP); IgA glomerulonephritis; Henoch-Schönlein purpura nephritis (HSPN); clinicopathological features

Submitted Sep 24, 2019. Accepted for publication Feb 09, 2020.

doi: 10.21037/apm.2020.04.04

View this article at: http://dx.doi.org/10.21037/apm.2020.04.04

(c) Annals of Palliative Medicine. All rights reserved. 


\section{Introduction}

Immunoglobulin A nephropathy (IgAN) is one of the most common types of glomerulonephritis, especially in the East Asia (1). In China, the proportion of IgAN in primary glomerular diseases has risen to $52.66 \%$ (2). HenochSchönlein purpura, in contrast, is considered as systemic small vessel vasculitis, and $70-80 \%$ of adult patients with Henoch-Schönlein purpura have developed nephritis with mesangial IgA deposits $(3,4)$. In China, HSPN has been the second-most common secondary glomerular diseases (2).

Both HSPN and IgAN have concomitant mesangial IgA deposits on pathology, suggesting that they share pathogenic mechanisms. The histological and immunofluorescent features are hard to distinguish between HSPN and IgAN (5). Several studies have revealed the differences in the clinical manifestations and outcomes between IgAN and HSPN $(6,7)$, which may be attributed to the mismatched age distribution and different prescriptions of steroids or other immunosuppressive drugs (8). Whether they are related is still under controversy.

It has been reported that adults have a higher incidence of IgAN, but the incidence of HSPN is relative low in adults. Therefore, fewer clinical studies have reported the renal outcomes in adult HSPN patients than in IgAN patients. Besides, the clinical manifestations of HSPN have not yet been fully elucidated in studies with large sample size, especially the HSPN of some special types.

The Oxford classification is based on five histopathologic features in pathological examination, and some studies have confirmed that Oxford classification is effective to predict the renal prognosis in IgAN patients (9). The morphologic lesions of HSPN have been proposed by the International Study Group of Kidney Disease in Childhood Pathology Classification, and other morphologic lesions are not taken into account such as interstitial inflammation, fibrosis, and tubular atrophy (10). Some clinical studies suggest it is feasible to apply the Oxford classification in patients with HSPN $(11,12)$. Among the parameters in Oxford classification, E score plays a minor role in predicting the prognosis of IgAN, but is associated with renal outcomes in adult HSPN patients (12).

Diffuse endocapillary proliferation (DEP), which involves $\geq 50 \%$ of all the glomeruli and $\geq 50 \%$ of the glomerular tuft is different from E1 (only endocapillary proliferation); DEP is rather frequent finding in the acute phase of poststreptococcal infection. However, it is rarely observed in both IgAN and HSPN, and the incidence is about $5 \%$ in $\operatorname{IgAN}$ and $2.17-4.05 \%$ in $\operatorname{HSPN}(13,14)$, and most of cases are reported in children. The present study aimed to clarify the differences and relationship in the clinicopathological features and outcomes between DEPIgAN and DEP-HSPN.

\section{Methods}

\section{Patients}

From January 2001 to April 2018, 2,689 adults were diagnosed with IgAN and 272 with HSPN in our renal center. Among them, 12 were diagnosed with DEP-IgAN (DEP-IgAN group) and 10 with DEP-HSPN (DEP-HSPN group) were enrolled into present study. The DEP was diagnosed when endocapillary hypercellularity involves $\geq 50 \%$ of all the glomeruli and the lesion involves $\geq 50 \%$ of the glomerular tuft. In addition, 24 IgAN patients without DEP (NDEP-IgAN group) and matched 20 HSPN patients without DEP (NDEP-HSPN group) were recruited. There were no marked differences in the baseline characteristics such as age, sex, and Oxford classification scores (M, S, T and C). Patients in both NDEP-IgAN group and NDEPHSPN group had complete clinical and pathological data (Figure 1).

All patients met the following criteria: (I) patients were $\geq 16$ years; (II) there were $>10$ glomeruli under light microscope; (III) IgAN patients (DEP-IgAN group and NDEP-IgAN group) had definite pathologic findings, and no sign of systemic involvement reminiscent of HSPN, systemic lupus erythematosus, or other systemic diseases; HSPN patients (DEP-HSPN group and NDEP-HSPN group) had no sign of other systemic diseases; (IV) Lightmicroscopy showed the lesions with DEP in both DEPIgAN group and DEP-HSPN group; (V) patients were followed up for more than 12 months.

The study was conducted according to the Declaration of Helsinki and approved by the Institutional Review Board of Hangzhou Hospital of Traditional Chinese medicine (No. H-1002-032-309). The informed consent was waived since the study did not infringe on patient privacy or health status.

\section{Data collection}

Clinical and demographic characteristics were collected from the medical records such as age, gender, medications, and blood pressure. Laboratory findings including 

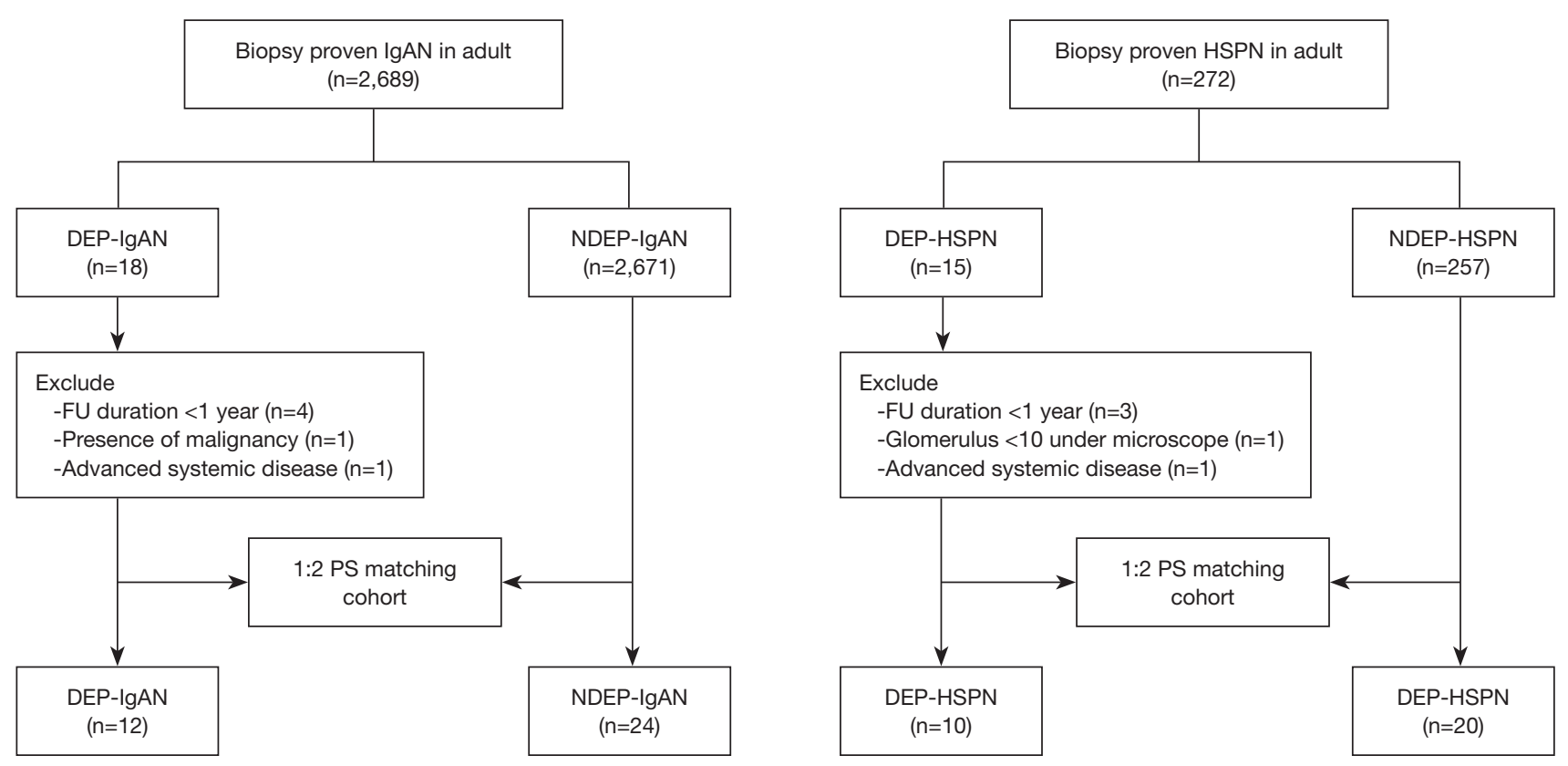

Figure 1 The flow diagram of patient recruitment. DEP, diffuse endocapillary proliferation; IgAN, immunoglobulin A nephropathy; HSPN, Henoch-Schönlein purpura nephritis.

24-hour urinary protein excretion, urinary erythrocyte count, serum creatinine, uric acid, urea nitrogen, albumin, total cholesterol concentrations, serum IgA level, and complement C3 level were also recorded. Renal survival was evaluated by a $>30 \%$ decline in the eGFR or the presence of end stage renal disease (ESRD). The eGFR was calculated using the four-variable Modification of Diet in Renal Disease formula (15).

\section{Histological parameters}

After renal biopsy, all the renal samples were evaluated and graded by two nephrologists according to the Oxford classification. In addition, for each histologic lesion, the degree of global glomerular sclerosis (GS), crescent ratio (CC), interstitial infiltration, renal vascular lesion (wall thickening and hyaline change) $(0-3$ or $0-4)$ were classified by using the Haas and Katafuchi system (16). The presence of endothelial swelling was also assessed.

The Oxford classification is based on the MEST-C score: mesangial hypercellularity ( $\mathrm{M} ; \mathrm{M} 0 \leq 50 \%$; $\mathrm{M} 1$ $>50 \%$;), endocapillary hypercellularity (E; E0: absence; $\mathrm{E} 1$ : presence), segmental sclerosis/adhesion (S; S0:absence; $\mathrm{S} 1$ :presence), tubular atrophy/interstitial fibrosis ( $\mathrm{T}$; T0 $\leq 25 \%$; T1: $26-50 \%$; T2: $>50 \%$ ), and cellular or fibrocellular crescents (C; C0: absence, $\mathrm{C} 1 \leq 25 \%$, and C2 > 25\%) (9).

\section{Treatment}

Renin-angiotensin system (RAS) blockers were used in patients with hypertension or urinary protein-to-creatinine ratio $\geq 0.5 \mathrm{mg} / \mathrm{d}$. Steroid treatment (prednisone 0.8 $1.0 \mathrm{mg} / \mathrm{kg} /$ day) was initiated in patients with persistent proteinuria $>3.0 \mathrm{~g} / \mathrm{d}$, unresponsive to RAS blockers or with evidence of severe active inflammation. Other immunosuppressive drugs, like cyclophosphamide and mycophenolate mofetil, were added when incomplete response or intolerance of steroid was present. Besides, for HSPN patients, steroid was also administered when there were evident systemic manifestations of vasculitis, even in the absence of proteinuria.

\section{Study outcomes}

The renal outcomes were graded as follows: Grade A (complete remission): normal renal function, without proteinuria or hematuria; Grade B (partial remission): persistent proteinuria $(<1.0 \mathrm{~g} / \mathrm{d}$ ), and/or hematuria ( $\geq 3 \mathrm{red}$ blood cells/high power field) without renal insufficiency; Grade C (no remission): persistent proteinuria ( $\geq 1.0 \mathrm{~g} / \mathrm{d}$ ), 
Table 1 Baseline clinical characteristics

\begin{tabular}{|c|c|c|c|c|c|c|c|}
\hline Indexes & \multicolumn{2}{|c|}{$\lg A N$} & \multicolumn{2}{|c|}{ HSPN } & $P_{A B}$ & $\mathrm{P}_{\mathrm{CD}}$ & $\mathrm{P}_{\mathrm{AC}}$ \\
\hline Age, years & $41.50(24,63.75)$ & $44.89(24.18,52.50)$ & $48.00(21.75,70.25)$ & $45(23.00,60.50)$ & 0.987 & 0.453 & 0.766 \\
\hline Male/Female & $8 / 4$ & $10 / 14$ & $3 / 7$ & $8 / 12$ & 0.157 & 0.702 & 0.087 \\
\hline Course, months & $2.00(0.35,5.50)$ & $9.50(1.00,21.00)$ & $1.00(0.28,2.25)$ & $5.50(1.17,11.75)$ & 0.043 & 0.006 & 0.259 \\
\hline Gross hematuria & $2(16.7 \%)$ & $13(54.2 \%)$ & $4(40.0 \%)$ & $1(5.0 \%)$ & 0.04 & 0.031 & 0.348 \\
\hline Proteinuria, g/day & $2.51(1.16,4.90)$ & $0.55(0.30,1.67)$ & $7.04(3.02,9.08)$ & $0.59(0.47,1.70)$ & 0.005 & 0.0001 & 0.018 \\
\hline Serum ALB, g/L & $32.10(27.58,34.15)$ & $37.5(33.75,41.53)$ & $24.75(19.70,27.33)$ & $36.4(31.8,39.78)$ & 0.004 & 0.0002 & 0.012 \\
\hline Serum creatinine, $\mu \mathrm{mol} / \mathrm{L}$ & $100.50(66.25,117.50)$ & $69.50(55.25,96.50)$ & $82.5(70.25,125.25)$ & $71(57,93)$ & 0.107 & 0.159 & 0.742 \\
\hline Serum C3, mg/dL & $84(75.45,88.48)$ & $82.90(65.25,95.50)$ & $92(60.75,103.50)$ & $99(85.75,116.75)$ & 0.626 & 0.153 & 0.372 \\
\hline
\end{tabular}

$P_{A B}$, compared with DEP-IgAN group and NDEP-IgAN group; $P_{C D}$, compared with DEP-HSPN group and NDEP-HSPN group; $P_{A C}$, compared with DEP-IgAN group and DEP-HSPN group. ALB, albumin; eGFR, estimated glomerular filtration rate; DEP, diffuse endocapillary proliferation; IgAN, immunoglobulin A nephropathy; HSPN, Henoch-Schönlein purpura nephritis.

and/or hematuria with moderate renal failure $(<30 \%$ decrease in the eGFR from the baseline); Grade D (renal failure): $\geq 30 \%$ decline in the eGFR from the baseline, ESRD or death. The primary endpoint was $\geq 30 \%$ decrease in eGFR from the baseline, ESRD, or death. For patients who lost to follow-up or died during the study period, data collected at the last follow up were used for analysis.

\section{Statistical analysis}

All statistical analyses were performed with SPSS version 23.0. Case control matching was conducted using the SAS system. Nonparametric variables are expressed as medians and interquartile ranges (IQRs) and compared with the Kruskal-Wallis test. Categorical variables are expressed as percentages and compared with the Pearson's Chi-square test or Fisher's exact test. Kaplan-Meier survival curves were used to analyze renal survival from the time of biopsy to the presence of endpoints. A value of $\mathrm{P}<0.05$ was considered statistically significant.

\section{Results}

\section{Clinical characteristics at baseline}

The baseline clinical characteristics are shown in Table 1 .
Results showed there were no marked differences in the median age at diagnosis, gender and course of disease between DEP-IgAN group and DEP-HSPN group. The age in both DEP-IgAN group and DEP-HSPN group displayed a bimodal-like distribution, and most of patients were younger than 30 years or older than 60 years (Figure 2).

The prevalence of hypertension and gross hematuria had no significant differences between DEP-IgAN group and DEP-HSPN group. The median proteinuria in the DEPHSPN group was 7.04 (IQR, 3.02-9.08) g/day, which was significantly higher than in the DEP-IgAN group [2.51 (IQR, 1.16-4.90) g/day; $\mathrm{P}=0.018]$. The median serum ALB in the DEP-HSPN group was 24.75 (IQR, 19.70-27.33) g/L, which was markedly lower than in the DEP-IgAN group [32.10 (IQR, 27.58-34.15) g/L, $\mathrm{P}=0.012$ ].

In the matched cohort of IgAN patients, the course of disease in DEP patients (DEP-IgAN group) was significantly shorter than in those patients without DEP (NDEP-IgAN group) [2.00 (IQR, 0.35-5.50) vs. 9.50 (IQR, 1.00-21.00) months, $\mathrm{P}=0.043]$. The clinical findings were worse in the DEP-IgAN group than in the NDEPIgAN group [gross hematuria: $16.67 \%$ vs. $54.17 \%$, $\mathrm{P}=0.04$; median proteinuria: 2.51 (IQR, 1.16-4.90) vs. 0.55 (IQR, 0.30-1.67) g/day, $\mathrm{P}=0.005$; median serum ALB: 32.10 (IQR, 27.58-34.15) vs. 37.50 (IQR, $33.75-$ 41.53) $\mathrm{g} / \mathrm{L}, \mathrm{P}=0.004]$. There were no marked differences 

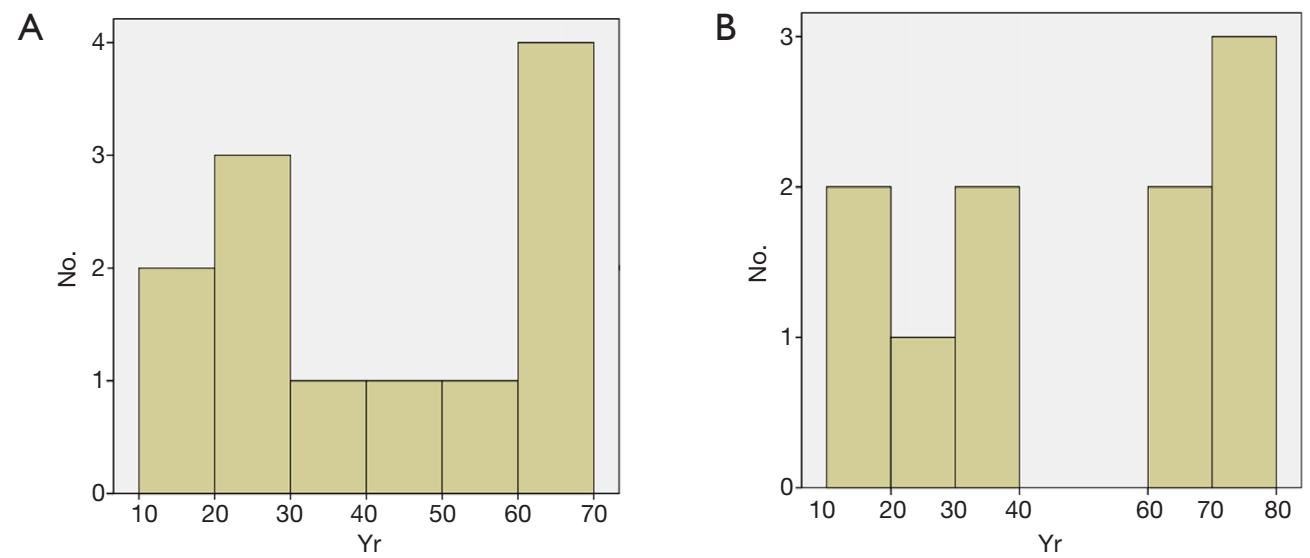

Figure 2 Age distribution of DEP-IgAN patients (A) and DEP-HSPN patients (B). Each histogram shows 10-year intervals. DEP, diffuse endocapillary proliferation; IgAN, immunoglobulin A nephropathy; HSPN, Henoch-Schönlein purpura nephritis.

in the hypertension, eGFR, serum creatinine, IgA and complement 3 between two groups.

In the matched cohort of HSPN patients, the course of disease in DEP patients (group DEP-HSPN) was significantly shorter than in those without DEP (NDEPHSPN group) [1.00 (IQR, 0.28-2.25) vs. 5.50 (IQR, $1.17-11.75$ ) months, $\mathrm{P}=0.006]$. The ratio of gross hematuria was significantly higher in the DEP-HSPN group than in the NDEP-HSPN group $(40.0 \%$ vs. $5.0 \%, \mathrm{P}=0.031)$. The clinical results were worse in the DEP-HSPN group [median proteinuria: 7.04 (IQR, 3.02-9.08) vs. 0.59 (IQR, 0.47-1.70) g/day, $\mathrm{P}=0.0001$; median serum ALB: 24.75 (IQR, 19.70-27.33) vs. 36.40 (IQR, 31.80-39.78) g/L, $\mathrm{P}=0.0002]$. Additionally, the eGFR was markedly lower in the DEP-HSPN group than in the NDEP-HSPN group [median eGFR: 64.3 (IQR, 48.85-81.53) vs. 94.95 (IQR, 62.75-116.88) $\mathrm{mL} / \mathrm{min}, \mathrm{P}=0.029]$. There were no marked differences in the hypertension, serum creatinine, IgA and complement 3 between two groups.

\section{Histological parameters}

The pathological characteristics are shown in Table 2. The S1 score of Oxford classification was observed in 8 of 12 (66.67\%) patients in the DEP-IgAN group, as compared to 2 of $10(20.0 \%)$ patients in the DEP-HSPN group $(\mathrm{P}=0.043)$. For renal vascular lesion, endothelial swelling was observed in 5 of $10(50.0 \%)$ patients in the DEPHSPN group, as compared to 1 of $12(8.3 \%)$ patients in the DEP-IgAN group $(\mathrm{P}=0.056)$. There were no significant differences in the $\mathrm{M}, \mathrm{T}$ and $\mathrm{C}$ scores of Oxford classification, and the grades of GS, CC and interstitial infiltration between DEP-IgAN group and DEP-HSPN group.

In the matched cohort, the incidence of endothelial swelling in renal vascular lesion was markedly higher in the DEP-HSPN group than in the NDEP-HSPN group $(50.0 \%$ vs. $10 \%, \mathrm{P}=0.026)$. There were no marked differences in the grades of GS, CC, interstitial infiltration, and renal vascular lesion between DEP-IgAN group and NDEP-IgAN group and between DEP-HSPN group and NDEP-HSPN group.

\section{Treatments and renal survival}

The treatments and renal survival are shown in Table 3. There were no significant difference in the steroids and other immunosuppressive drugs used between DEPIgAN group and DEP-HSPN group, between DEP-IgAN group and NDEP-IgAN group, and between DEP-HSPN group and NDEP-HSPN group. In the DEP-HSPN group, $2(20 \%)$ patients showed no clinical remission, $3(30 \%)$ developed ESRD, and the renal outcome was significantly poorer in the DEP-HSPN group than in the DEP-IgAN group and NDEP-HSPN group $(\mathrm{P}=0.015$ and 0.010, respectively). The Kaplan-Meier survival curves are showed in Figure 3. The cumulative renal survival rate in the NDEP-IgAN group at 3 and 6 years was $95.7 \%$ and $79 \%$, respectively. The cumulative renal survival rate in the DEP-HSPN group at 4 and 6 years was $77.1 \%$ and $53.3 \%$, respectively. The endpoint events were not observed in the DEP-IgAN group and NDEP-HSPN group. In our cohort, 
Table 2 Pathological characteristics

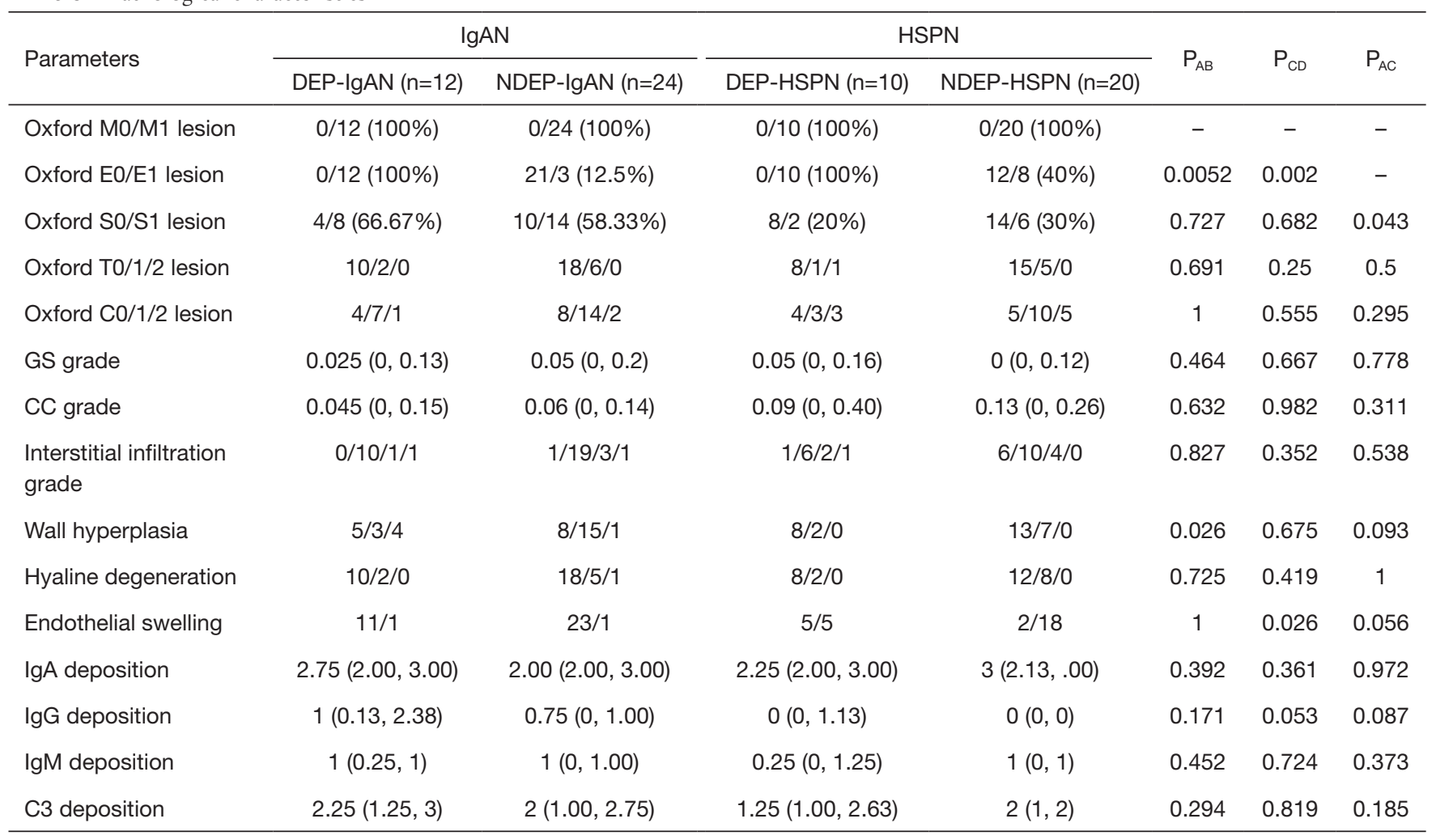

$\mathrm{P}_{\mathrm{AB}}$, compared with DEP-IgAN group and NDEP-IgAN group; $\mathrm{P}_{\mathrm{CD}}$, compared with DEP-HSPN group and NDEP-HSPN group; $\mathrm{P}_{\mathrm{AC}}$, compared with DEP-IgAN group and DEP-HSPN group. M, mesangial hypercellularity; E, endocapillary proliferation; S, segmental sclerosis/adhesion; T, tubular atrophy/interstitial fibrosis; C cellular or fibrocellular crescents; GS, global glomerular sclerosis, CC, crescent ratio; DEP, diffuse endocapillary proliferation; IgAN, immunoglobulin A nephropathy; HSPN, Henoch-Schönlein purpura nephritis.

Table 3 Treatments and renal survival

\begin{tabular}{|c|c|c|c|c|c|c|c|}
\hline Indexes & \multicolumn{2}{|c|}{$\lg A N$} & \multicolumn{2}{|c|}{ HSPN } & $P_{A B}$ & $\mathrm{P}_{\mathrm{CD}}$ & $\mathrm{P}_{\mathrm{AC}}$ \\
\hline \multicolumn{8}{|l|}{ Treatments } \\
\hline RAS blockers & $6(50.0 \%)$ & $20(83.3 \%)$ & $2(20.0 \%)$ & $13(65.0 \%)$ & 0.053 & 0.050 & 0.204 \\
\hline Steroid & $10(83.3 \%)$ & $15(62.5 \%)$ & $10(100 \%)$ & $17(85.0 \%)$ & 0.268 & 0.532 & 0.481 \\
\hline Fellow-up months & $41.50(15.75,92.75)$ & $66(51.00,77.75)$ & $49(18.75,76.25)$ & $41(30.50,71.00)$ & 0.150 & 0.910 & 0.843 \\
\hline Renal outcomes(A/B/C/D) & $8 / 1 / 3 / 0$ & $17 / 3 / 1 / 3$ & $1 / 4 / 2 / 3$ & $12 / 7 / 1 / 0$ & 0.869 & 0.010 & 0.015 \\
\hline Primary endpoint & $0 / 12$ & $3 / 24$ & $3 / 10$ & $0 / 20$ & 0.285 & 0.103 & 0.214 \\
\hline
\end{tabular}

$\mathrm{P}_{\mathrm{AB}}$, compared with DEP-IgAN group and NDEP-IgAN group; $\mathrm{P}_{\mathrm{CD}}$, compared with DEP-HSPN group and NDEP-HSPN group; $\mathrm{P}_{\mathrm{AC}}$, compared with DEP-IgAN group and DEP-HSPN group. RAS, renin-angiotensin system. DEP, diffuse endocapillary proliferation; IgAN, immunoglobulin A nephropathy; HSPN, Henoch-Schönlein purpura nephritis. 


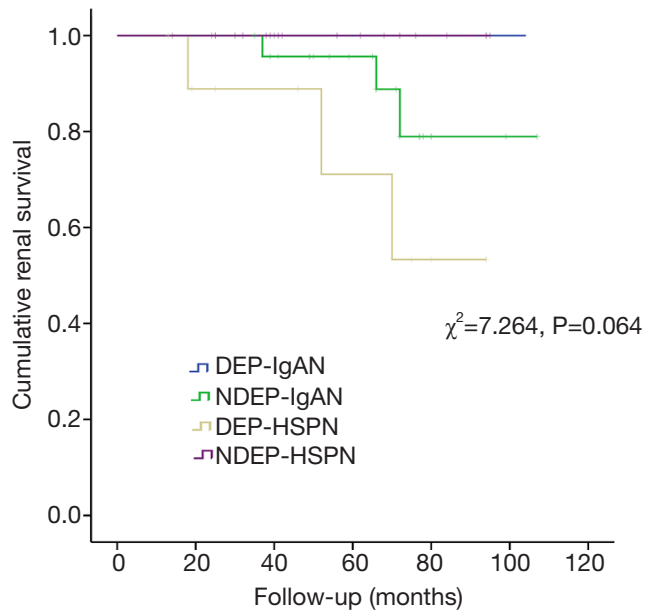

Figure 3 Kaplan-Meier renal survival analysis in four groups. DEP, diffuse endocapillary proliferation; IgAN, immunoglobulin A nephropathy; HSPN, Henoch-Schönlein purpura nephritis.

Kaplan-Meier analysis revealed no significant difference in the cumulative renal survival among four groups (log rank $\chi^{2}=7.264, \mathrm{P}=0.064$ ) (Figure 2). There was no marked difference in the cumulative renal survival between DEPIgAN group and DEP-HSPN group $\left(\log\right.$ rank $\chi^{2}=2.518$, $\mathrm{P}=0.113$ ), and between DEP-IgAN group and NDEPIgAN group ( $\left.\log \operatorname{rank} \chi^{2}=0.763, \mathrm{P}=0.382\right)$. However, the renal cumulative survival rate was significantly lower in the DEP-HSPN group than in the NDEP-HSPN group (log $\left.\operatorname{rank} \chi^{2}=4.875, \mathrm{P}=0.027\right)$.

\section{Discussion}

Glomerular capillary endothelial cells belong to the endothelial system but are functionally different from other endothelial cells due to their special anatomical location. As a part of the renal filtration barrier, they play important roles in maintaining the integrity of glomerular capillary structure and selective filtration barrier function. They are regulated by a variety of substances in the plasma and podocytes, and can affect the negative charge barrier of the glomerular basement membrane (17).

Endocapillary hypercellularity is often observed in the histological lesion of IgAN and HSPN. In addition, the lack of terminal b1-galactosyl residues in the hinge region of IgA1 has been observed in both diseases, along with the increased aberrantly glycosylated IgA1 $(5,18)$. However, the deposition of IgA component or other complements in the capillary wall is not necessary for the progression of IgAN with EP. In 2014, Hodgin et al. (19) evaluated the glomerular transcriptome of microdissected kidney biopsies from IgAN patients with EP, and identified a total of 424 differentially expressed genes between EPIgAN patients and NEP-IgAN patients. Moreover, most of these differentially expressed genes are related to the inflammation and $\mathrm{T}$ cell signal transduction, which suggests that IgAN may mediate the occurrence of EP through a similar mechanism. In HSPN, it has been reported that the activation of endothelial nitric oxide synthase and the enhanced binding of IgA1 anti-endothelial cell antibodies (AECA) to the endothelial cells contribute to the lesions of endocapillary proliferation $(20,21)$.

In the present study, there was no significant difference in the median age of diagnosis between DEP-IgAN group and DEP-HSPN group. The age in both DEP groups seemed to display a bimodal-like distribution, and most of patients were younger than 30 years or older than 60 years (Figure 2). The age distribution was similar to that in HSPN patients with $\mathrm{E}$ lesions as reported in a previous study (6). In addition, the course of disease was markedly shorter in the DEP groups, especially in the DEP-HSPN group, which suggests DEP being an acute lesion.

Some studies, including the Oxford cohort and the European VALGA cohort, have shown the correlation between capillary endothelial proliferative lesion and baseline urinary protein level in patients with IgAN $(22,23)$. In another cohort study on IgAN patients with DEP, the average proteinuria was $6.9 \mathrm{~g} / \mathrm{d}$, which was significantly higher than in IgAN patients without proliferative endocapillary lesions (13). Our study also showed an increased proteinuria in two DEP groups, and the proteinuria in patients with DEP-HSPN was higher than in those with DEP-IgAN, which may be related to the extra involvement of small vasculitis and the endothelial injury caused by AECA and other factors. No evidence supports that the renal function in DEP-IgAN patients was significantly different from that in patients with other types of IgAN. However, in the present study, the baseline eGFR was markedly different between DEP-HSPN group and NDEP-HSPN group, though the serum creatinine was comparable, which was attributed to the relatively lower plasma ALB affecting the calculation of eGFR in the DEPHSPN group.

The $\mathrm{M}, \mathrm{T}$ and $\mathrm{C}$ scores were comparable between DEP-HSPN group and DEP-IgAN group, but patients with DEP-HSPN had lower S1 scores, suggesting milder segmental glomerulosclerosis. Moreover, DEP-HSPN 
patients had more severe capillary swelling. As a kind of vasculitis, the main pathological changes of purpura are the broken white blood cells in the superficial dermis, swelling of vascular endothelial cells and infiltration of neutrophils (24). The injury of endothelial cells can be manifested as cell swelling at early stage, which indicates the lesion being mainly acute. This can be explained as the short course of DEP-HSPN. Therefore, pathological changes such as focal segmental sclerosis or vascular wall hyperplasia are rare at this stage.

$\mathrm{E}$ score plays a secondary role in the prognosis of IgAN. $\mathrm{Lv}$ et al. (25) investigated 16 retrospective studies between 2009 and 2012, involving 3,893 patients. Multivariate analysis showed no significant correlation between E1 and IgAN prognosis. However, Inagaki et al. (12) reported that EP was associated with the renal prognosis in adult patients with HSPN, regardless the application of immunosuppressive drugs. As for the predictive value of DEP, DEP showed inconsistent effects on the treatment and prognosis of IgAN. D'amico et al. (26) found the risk for renal failure in IgAN patients with DEP was significantly higher than in those with mild or without endocapillary proliferation. Liu et al. found there was no significant difference between IgAN patients with DEP and IgAN patients without DEP. DEP-IgAN patients had normal renal function are more likely to achieve a better prognosis (13). In our study, considerably more patients with DEP-IgAN or DEP-HSPN were treated with steroids, as the proteinuria in the DEP groups was significantly higher. Patients in the DEP-IgAN group achieved a better therapeutic efficacy and had a good prognosis. However, the therapeutic efficacy in the DEP-HSPN group was poorer than in the NDEP-HSPN group and DEP-IgAN group. Even in the presence of active immunosuppressive treatment, complete remission was found in only one patient, and 3 patients with DEP developed endpoint events, indicating that HSPN patients with DEP are more resistant to immunosuppressive therapy than DEP-IgAN patients. Log-rank test also indicated that patients in the DEP-HSPN group had lower renal cumulative survival rate as compared to NDEP-HSPN patients in which there were 8 patients with $\mathrm{E} 1$ lesion.

Proliferative lesion in the capillaries may disappear when repeated renal biopsy is performed in IgAN patients with diffuse capillary hyperplasia, indicating that this type of lesion is reversible (27). Different from IgAN, in HSPN patients, IgA1 AECA binds to small vessels via $\beta 2$-glycoprotein I ( $\beta 2 \mathrm{GPI}$ ) or other factors on the endothelial cells to induce the production of inflammatory cytokines. Processes such as antibody-dependent cellular cytotoxicity (ADCC), complement dependent cytotoxicity (CDC), neutrophil extracellular traps (NETs) and reactive oxygen species (ROS) production may cause damage to the endothelial cells (20). Furthermore, inflammatory waterfall and feedback effect may cause continuous and irreversible damage. Therefore, our findings suggest the significance of DEP in the IgAN and HSPN is different, which reminds clinicians to pay more attention to HSPN patients with DEP.

There were still limitations in this study. This was a retrospective study from a single center, and the sample size was small. Although the incidence of HSPN is higher in Asian people than in other populations, adult HSPN is still rare among secondary kidney diseases, especially HSPN with DEP. Therefore, it is difficult to enroll a sufficient number of adult patients with HSPN. Thus, more prospective, multi-center studies with large sample size are needed to confirm our findings.

\section{Conclusions}

The DEP is significantly associated with more severe proteinuria and hematuria in both IgAN and HSPN. Different from DEP-IgAN, HSPN-DEP patients have poorer response to therapy and poorer renal outcome, even under active immunosuppressive therapy.

\section{Acknowledgments}

Funding: None.

\section{Footnote}

Conflicts of Interest: All authors have completed the ICMJE uniform disclosure form (available at http://dx.doi. org/10.21037/apm.2020.04.04). The authors have no conflicts of interest to declare.

Ethical Statement: The authors are accountable for all aspects of the work in ensuring that questions related to the accuracy or integrity of any part of the work are appropriately investigated and resolved. The study was conducted according to the Declaration of Helsinki and approved by the Institutional Review Board of Hangzhou Hospital of Traditional Chinese Medicine (No. H-1002032-309). The informed consent was waived since the study did not infringe on patient privacy or health status. 
Open Access Statement: This is an Open Access article distributed in accordance with the Creative Commons Attribution-NonCommercial-NoDerivs 4.0 International License (CC BY-NC-ND 4.0), which permits the noncommercial replication and distribution of the article with the strict proviso that no changes or edits are made and the original work is properly cited (including links to both the formal publication through the relevant DOI and the license). See: https://creativecommons.org/licenses/by-nc-nd/4.0/.

\section{References}

1. D'Amico G. Natural history of idiopathic IgA nephropathy and factors predictive of disease outcome. Semin Nephrol 2004;24:179-96.

2. Hou JH, Zhu HX, Zhou ML, et al. Changes in the Spectrum of Kidney Diseases: An Analysis of 40,759 Biopsy-Proven Cases from 2003 to 2014 in China. Kidney Dis (Basel) 2018;4:10-9.

3. Kang Y, Park JS, Ha YJ, et al. Differences in clinical manifestations and outcomes between adult and child patients with Henoch-Schonlein purpura. J Korean Med Sci 2014;29:198-203.

4. Hong S, Ahn SM, Lim DH, et al. Late-onset IgA vasculitis in adult patients exhibits distinct clinical characteristics and outcomes. Clin Exp Rheumatol 2016;34:S77-83.

5. Davin JC, Ten Berge IJ, Weening JJ. What is the difference between IgA nephropathy and Henoch-Schonlein purpura nephritis? Kidney Int 2001;59:823-34.

6. Komatsu H, Fujimoto S, Yoshikawa N, et al. Clinical manifestations of Henoch-Schonlein purpura nephritis and IgA nephropathy: comparative analysis of data from the Japan Renal Biopsy Registry (J-RBR). Clin Exp Nephrol 2016;20:552-60.

7. Calvo-Río V, Loricera J, Martin L, et al. HenochSchonlein purpura nephritis and IgA nephropathy: a comparative clinical study. Clin Exp Rheumatol 2013;31:S45-51.

8. Oh HJ, Ahn SV, Yoo DE, et al. Clinical outcomes, when matched at presentation, do not vary between adultonset Henoch-Schonlein purpura nephritis and IgA nephropathy. Kidney Int 2012;82:1304-12.

9. Trimarchi H, Barratt J, Cattran DC, et al. Oxford Classification of IgA nephropathy 2016: an update from the IgA Nephropathy Classification Working Group. Kidney Int 2017;91:1014-21.

10. Davin JC, Coppo R. Pitfalls in recommending evidence-based guidelines for a protean disease like
Henoch-Schonlein purpura nephritis. Pediatr Nephrol 2013;28:1897-903.

11. Kim CH, Lim BJ, Bae YS, et al. Using the Oxford classification of IgA nephropathy to predict long-term outcomes of Henoch-Schonlein purpura nephritis in adults. Mod Pathol 2014;27:972-82.

12. Inagaki $\mathrm{K}$, Kaihan $\mathrm{AB}$, Hachiya $\mathrm{A}$, et al. Clinical impact of endocapillary proliferation according to the Oxford classification among adults with Henoch-Schonlein purpura nephritis: a multicenter retrospective cohort study. BMC Nephrol 2018;19:208.

13. Liu LJ, Li GT, Zhou Y, et al. Clinicopathologic features and outcomes in endocapillary proliferative IgA nephropathy. Nephron Clin Pract 2010;115:c161-7.

14. Fang X, Yi Z, Dang X. Clinical analysis of 236 children with Henoch-Schonlein purpura. Journal of Clinical Pediatrics 2006;24:46-9.

15. National Kidney Foundation. K/DOQI clinical practice guidelines for chronic kidney disease: evaluation, classification, and stratification. Am J Kidney Dis 2002;39:S1-266.

16. Haas M. Histologic subclassification of IgA nephropathy: a clinicopathologic study of 244 cases. Am J Kidney Dis 1997;29:829-42.

17. Fogo $A B$, Kon $V$. The glomerulus--a view from the inside--the endothelial cell. Int J Biochem Cell Biol 2010;42:1388-97.

18. Novak J, Moldoveanu Z, Renfrow MB, et al. IgA nephropathy and Henoch-Schoenlein purpura nephritis: aberrant glycosylation of IgA1, formation of IgA1containing immune complexes, and activation of mesangial cells. Contrib Nephrol 2007;157:134-8.

19. Hodgin JB, Berthier CC, John R, et al. The molecular phenotype of endocapillary proliferation: novel therapeutic targets for IgA nephropathy. PLoS One 2014;9:e103413.

20. Heineke $\mathrm{MH}$, Ballering AV, Jamin A, et al. New insights in the pathogenesis of immunoglobulin A vasculitis (HenochSchonlein purpura). Autoimmun Rev 2017;16:1246-53.

21. Yang YH, Huang YH, Lin YL, et al. Circulating IgA from acute stage of childhood Henoch-Schonlein purpura can enhance endothelial interleukin (IL)-8 production through MEK/ERK signalling pathway. Clin Exp Immunol 2006;144:247-53.

22. Coppo R, Troyanov S, Bellur S, et al. Validation of the Oxford classification of IgA nephropathy in cohorts with different presentations and treatments. Kidney Int 2014;86:828-36. 
23. Zeng $\mathrm{CH}$, Le $\mathrm{W}, \mathrm{Ni} Z$, et al. A multicenter application and evaluation of the oxford classification of IgA nephropathy in adult Chinese patients. Am J Kidney Dis 2012;60:812-20.

24. Yang YH, Yu HH, Chiang BL. The diagnosis and classification of Henoch-Schonlein purpura: an updated review. Autoimmun Rev 2014;13:355-8.

25. Lv J, Shi S, Xu D, et al. Evaluation of the Oxford Classification of IgA nephropathy: a systematic review and meta-analysis. Am J Kidney Dis 2013;62:891-9.

26. D'Amico G, Minetti L, Ponticelli C, et al. Prognostic indicators in idiopathic IgA mesangial nephropathy. Q J Med 1986;59:363-78.

27. Shen XH, Liang SS, Chen HM, et al. Reversal of active glomerular lesions after immunosuppressive therapy in patients with IgA nephropathy: a repeat-biopsy based observation. J Nephrol 2015;28:441-9.
Cite this article as: Yu J, Ni X, Ni J, Jiang F, Yu D, Zhou L. Comparison of clinicopathological features and prognosis between IgA nephropathy and purpura nephritis in adults with diffuse endocapillary proliferation: a single-center cohort study. Ann Palliat Med 2020;9(3):795-804. doi: 10.21037/ apm.2020.04.04 